\title{
Increased Capillary Branching Contributes to Angiotensin Type 1 Receptor Blocker (ARB)-Induced Regression of Sclerosis
}

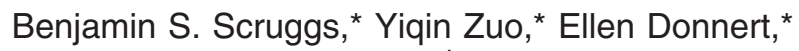 \\ Lijun Ma, ${ }^{*}$ John F. Bertram, ${ }^{\dagger}$ and Agnes B. Fogo* \\ From the Department of Pathology, Vanderbilt University \\ Medical Center, Nashville, Tennessee; and the Department of \\ Anatomy \& Developmental Biology, ${ }^{\dagger}$ Monash University, Victoria, \\ Australia
}

Chronic kidney disease is characterized by progressive glomerulosclerosis and tubulointerstitial fibrosis. High-dose angiotensin type 1 receptor blocker (ARB) or angiotensin-converting enzyme inhibitor can induce regression of existing glomerulosclerosis, at least in part by decreasing matrix accumulation. However, the potential mechanisms of remodeling of capillary loops remain obscure. This study aimed to determine whether capillary branching was augmented in glomeruli with ARB-induced regression of sclerosis. Three-dimensional confocal images were assessed by graph theory analysis to explore the topology of the glomerular capillary network. Compared with normal glomeruli, glomeruli of rats with progressive sclerosis were enlarged but had a significantly reduced number of capillary segments and capillary branch points and decreased complexity of the glomerular network. In contrast, in rats with regression of sclerosis induced by ARB, glomerular enlargement was due to a significantly increased number of glomerular capillary segments and capillary branch points and restored complexity of the capillary network. These data support the theory that capillary growth contributes to regression of sclerosis and is mediated at least in part by ARB-induced increased complexity and branching of capillary segments. (Am J Patbol 2011, 178:1891-1898; DOI: 10.1016/j.ajpath.2010.12.029)

Chronic kidney disease (CKD), no matter what the primary cause, ultimately is manifest by progressive glomerulosclerosis and tubulointerstitial fibrosis. Current strategies for treating CKD aim to delay end-stage renal disease by optimizing blood pressure control and de- creasing proteinuria. Recent experimental approaches have begun to examine the possibility of regression of glomerulosclerosis, as recently reviewed. ${ }^{1,2}$ Proof of concept of regression was demonstrated in patients with diabetic nephropathy whose diabetes was cured by pancreas transplantation, with regression of glomerulosclerosis and tubulointerstitial lesions during the next 10 years. ${ }^{3}$ However, the specific cellular mechanisms underlying the decreased sclerosis ensuing after cure of the pathogenic insult of diabetes could not be shown in these human biopsy studies. The renin-angiotensin-aldosterone system has also been the focus of many interventions to modulate CKD beyond blood pressure control, especially when the initial disease process cannot be eliminated. We and others have shown in experimental models that high-dose angiotensin type 1 receptor blocker (ARB) or angiotensin-converting enzyme inhibitor (ACEI) beyond levels required to control systemic and capillary blood pressure can induce regression of existing sclerosis. ${ }^{4-10}$ Additional benefit to ameliorate or even regress sclerosis or progressive CKD clinically in experimental models and in human CKD has been achieved with combination of angiotensin blockade and a statin, pointing to the complexity of the mechanisms involved. ${ }^{11}$

Currently, the mechanisms through which regression could occur are not well understood. New glomeruli cannot be generated after term birth in humans, but segmentally scarred glomeruli could potentially undergo regression of existing sclerosis by resorption of areas with matrix accumulation and enhanced growth of the remaining capillary loops. ${ }^{10,12}$ Our previous data have shown that such ARBinduced improvement in sclerosis was indeed linked to less matrix accumulation in glomeruli, with an associated decrease in plasminogen activator inhibitor-1 (PAI-1) and increased plasmin activity. ${ }^{4} \mathrm{PAl}-1$ is the major inhibitor of the

Supported by National Institutes of Health, National Institute of Diabetes and Digestive and Kidney Diseases grant 56942.

B.S.S. and Y.Z. contributed equally to this work.

Accepted for publication December 23, 2010.

Address reprint requests to Agnes B. Fogo, Medical Center North C-3310, Department of Pathology, Vanderbilt University Medical Center, Nashville, TN 37232. E-mail: agnes.fogo@vanderbilt.edu. 
plasmin/plasminogen activator system and inhibits both fibrinolysis and proteolysis. ${ }^{11}$ PAl-1 also has plasmin-independent effects on matrix and cell migration. PAI-1 is induced by many profibrotic stimuli, including angiotensin type 2 through the angiotensin type 1 receptor and transforming growth factor- $\beta .{ }^{13}$ Our previous data focused on modulation of matrix accumulation as a mechanism that contributes to regression of sclerosis. ${ }^{4}$ Clearly, additional benefit could be achieved by not only reducing matrix but also augmenting capillary growth in remaining nonsclerosed segments of glomeruli. Interestingly, previous studies by Adamczak et $\mathrm{al}^{10}$ observed fewer capillaries per glomerulus in a model of regression induced by ACEI. Our in vitro data in cultured glomerular endothelial cells suggest that capillary growth could be achieved with high-dose ARB, perhaps contributed to by improved podocyte survival induced by ARB and/or effects directly on the endothelium. ${ }^{14}$ We therefore aimed to determine whether this mechanism of angiogenesis was present in vivo in ARB-induced regression of sclerosis. Three-dimensional confocal images were analyzed by graph theory analysis to explore the branching pattern of the capillary network in normal glomeruli versus glomeruli with sclerosis induced by 5/6 nephrectomy and compared with glomeruli in which ARB induced regression of sclerosis.

\section{Material and Methods}

\section{Animals}

Glomerulosclerosis was induced by $5 / 6$ nephrectomy in adult male Sprague Dawley rats (250 to 300 g; Charles River, Wilmington, MA). Briefly, with the rats under pentobarbital anesthesia, the right kidney was removed, and branches of the left kidney were ligated to induce 5/6 renal ablation. This model induces mild proteinuria, hypertension, and sclerosis by 4 weeks, with well-established sclerosis and worsened hypertension and sclerosis by week 8 and ongoing progressive sclerosis and death from uremia occurring in untreated 5/6 nephrectomy rats beyond 12 weeks. ${ }^{4}$ Serum creatinine levels are increased at the time of 5/6 nephrectomy due to removal of the renal mass, with a progressive decrease in glomerular filtration rate as CKD worsens. ${ }^{4}$ Rats were housed under conditions of a 12-hour light/dark cycle at $70^{\circ} \mathrm{F}$ with $40 \%$ humidity and 12 air exchanges per hour and received normal rat chow and water ad libitum (5001 diet, Purina Laboratory Rodent diet, $23.4 \%$ protein, $4.5 \%$ fat, $6.0 \%$ fiber, $0.4 \%$ sodium). At 8 weeks, sclerosis was established by open renal biopsy, yielding on average 25 glomeruli, as previously reported. ${ }^{4}$ Rats were then divided into groups with an equal starting point of sclerosis in these biopsy specimens as previously reported. A subset of rats was then treated from week 8 to 12 with ARB (losartan, $80 \mathrm{mg} / \mathrm{L}$ drinking water; equivalent to 10 $\mathrm{mg} / \mathrm{kg}$ body weight; and fourfold usual antihypertensive dose) and compared with untreated 5/6 nephrectomy rats, with matched biopsy levels of sclerosis. All 5/6 nephrectomy animals were sacrificed at 12 weeks. ${ }^{4}$ Normal kidney analysis was performed from kidneys removed at the time of the 5/6 nephrectomy. Animal care and procedures were performed in accordance with National Institutes of Health and Vanderbilt University animal care facility guidelines. Results from these studies have been previously reported, ${ }^{4}$ showing that regression occurred in $62 \%$ of ARB-treated rats, contrasting progressive sclerosis in untreated $5 / 6$ nephrectomy controls, assessed by analyzing biopsy sclerosis and comparing with autopsy sclerosis by light microscopic assessment in the same animals. We then chose a representative subset of rats from those previously reported studies that had adequate remaining tissue from autopsy for further analyses. We selected rats from that previous study with adequate additional tissue remaining from the autopsy that showed biopsy-proven regression of sclerosis in response to ARB treatment $(n=3)$ and compared them with rats with no treatment after 5/6 nephrectomy that had biopsy-proven progression of sclerosis $(n=3)$. We then performed in-depth confocal analysis of tissue from 12 weeks after $5 / 6$ nephrectomy and compared the results with normal kidneys $(n=3)$.

\section{Tissue Preparation}

On the basis of our previous data, glomeruli at 12 weeks after 5/6 nephrectomy in the rat range from 8000 to approximately $28,000 \mu \mathrm{m}^{2}$ in maximal planar cross-sectional area, on average approximately 15,000, corresponding to an average diameter range of approximately 100 to $110 \mu \mathrm{m}$ up to approximately 180 to $190 \mu \mathrm{m} .{ }^{15,16}$ We chose the maximal thickness section technically possible to maintain optimal permeabilization and staining throughout most of the depth of the tissue and also imaged most of a glomerular tuft. Our analysis of image quality through the z-stacks confirmed that sections through, on average, $65 \mu \mathrm{m}$ maintained adequate brightness of images (range, 58-75 $\mu \mathrm{m}$ ). Tissue was therefore sectioned at $100 \mu \mathrm{m}$ on a freezing microtome (model 860; American Optical Company, Southbridge, MA) to encompass as much as possible of the glomerular tufts and allow for maximal imaging. The frozen section was then removed to a Petri dish and incubated in cold $4 \%$ paraformaldehyde on ice for 20 minutes. Tissue sections were then permeabilized by incubating in $0.1 \%$ Triton $\mathrm{X}-100$ at $4^{\circ} \mathrm{C}$ for at least 8 hours. Rinses, blocking, and antibody incubations were performed for 8 to 12 hours each on a rocker plate at $4^{\circ} \mathrm{C}$. Tissue was stained with rabbit anti-vimentin for visualization of podocytes (1:100; Dakocytomation, Carpinteria, CA) and labeled with fluorescein isothiocyanate-conjugated goat anti-rabbit (1: 200; Jackson ImmunoResearch Laboratories, West Grove, PA; diluted in $2 \%$ bovine serum albumin and $0.1 \%$ Triton X-100 in PBS). Sections were subsequently double-stained with rhodamine-labeled antilens culinaris agglutinin (1:200; Vector Labs, Burlingame, CA) to stain basement membranes and matrix to allow visualization of capillary loops. Stained sections were placed in glass bottom No. 1.5 culture dishes (MatTek Corp., Ashland, $\mathrm{MA}$ ) and held in place with a coverslip for confocal microscopy. 


\section{Confocal Microscopy}

Glomeruli were randomly selected from each animal for z-step imaging, with these images capturing most of the glomerular tuft in most glomeruli studied. Z-step glomerular images were collected using a Zeiss LSM510 confocal microscope with a $\times 40$ oil immersion, 1.3-NA objective (Carl Zeiss Microlmaging Inc., Thornwood, NY) at the Cell Imaging Shared Resource Center at Vanderbilt Medical Center, Nashville, TN. The thickness of individual coverslips was measured by a micrometer and the objective adjusted accordingly before imaging of each sample. The collection parameters for confocal images were identical in all specimens with a spacing of $0.5 \mu \mathrm{m}$, zoom of 1.07 , pinhole size of $80 \mu \mathrm{m}$, and the laser set at $50 \%$.

\section{Morphological and Statistical Analysis}

Glomerular volume was calculated from a stack of images taken at incremental z-steps through a glomerulus, using Metamorph software (Molecular Devices, Inc., Sunnyvale, CA) for calculations. Using the center image of each stack as a reference, the area of interest was defined along with the threshold for the stack. Distances for the $\times 60$ magnification images were calibrated by converting 2.481 pixels to $1 \mu \mathrm{m}$. The measured volume function was then used to calculate the volume of a glomerulus via the image stack.

To determine the length of capillaries in a glomerulus $\left(L_{\text {cap,glom }}\right)$, the total number of capillary profiles in complete glomerular profiles was determined by counting the number of capillary segment profiles in five evenly spaced optical sections through the glomerulus, thus ensuring that no capillary was measured more than once. The length density of capillaries [ie, length per unit volume $\left.\left(L_{V \text { cap,glom }}\right)\right]$ was calculated using the following formula:

$$
\mathrm{L}_{\text {Vcap,glom }}=(2 \times Q) / A_{\text {glom }}
$$

where $Q$ is the number of capillary profiles within the glomerulus and $\mathrm{A}_{\mathrm{glom}}$ is the area of glomerular tuft analyzed.

$L_{\text {cap,glom }}$ was calculated by multiplying $L_{V_{c a p} \text {,glom }}$ by glomerular volume as follows:

$$
\mathrm{L}_{\text {cap,glom }}=\mathrm{L}_{\text {Vcap,glom }} \times \mathrm{V}_{\text {glom }}
$$

The total number of capillary segments in a glomerulus was estimated using an unbiased method based on the Euler-Poincaré characteristic. The Euler number is a measurement of the connectivity in a network. When a new capillary is formed, a new loop is created in the capillary network. This new capillary loop is then reflected in the Euler number by an increase of one unit because the connectivity has increased. The Euler number can be estimated by analyzing topological events of capillary lumina. These topological events are observed by comparing capillary lumina across a physical dissector. Two physical dissectors were used to estimate the changes in capillary lumina in confocal z-sections above and below a central section. The luminal events compared be- tween sections are the appearance of a capillary lumen (a luminal fragment), the appearance of an isolated island of capillary wall inside the lumen of a capillary profile (a luminal lagoon), and the division of a capillary lumen into two or more capillary lumina (a luminal connection). The Euler number was calculated using the following formula:

Euler number $=\Pi(n$ fragments $+n$ lagoons

$-n$ connections)

The $\Pi$ is included in this equation because each topologically defined capillary has two luminal connections.

The numerical capillary density $\left(\mathrm{W}_{\mathrm{Vcap}, \mathrm{glom}}\right)$ was then calculated using the following formula:

$$
\mathrm{W}_{\text {Vcap,glom }}=(\mathrm{cap}) /\left(2 \times \mathrm{t} \times \mathrm{A}_{\text {glom }}\right)
$$

where $t$ is section thickness, $A_{\text {glom }}$ is the glomerular area analyzed, and 2 accounts for a physical dissector in both the positive and negative directions from the central section.

The total number of capillaries per glomerulus ( $w_{\text {cap,glom }}$ ) was determined using the following formula:

$$
\mathrm{W}_{\text {cap }, \text { glom }}=\left(\mathrm{V}_{\text {glom }} \times \mathrm{W}_{\text {Vcap,glom }}\right)+2
$$

A

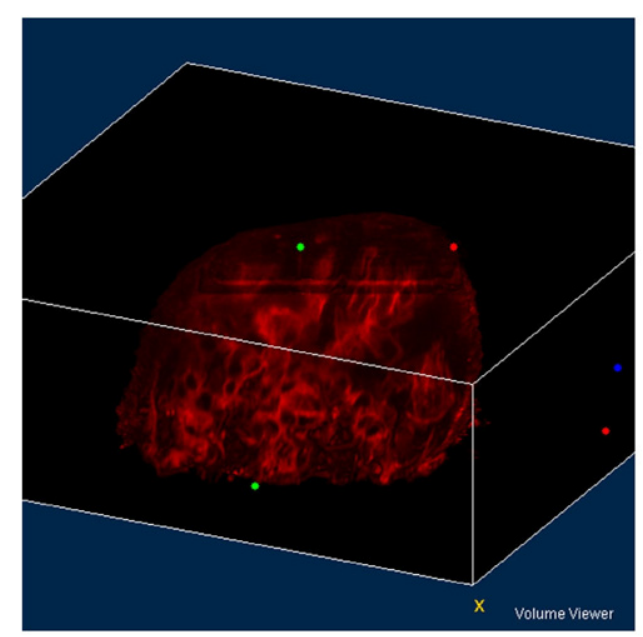

B

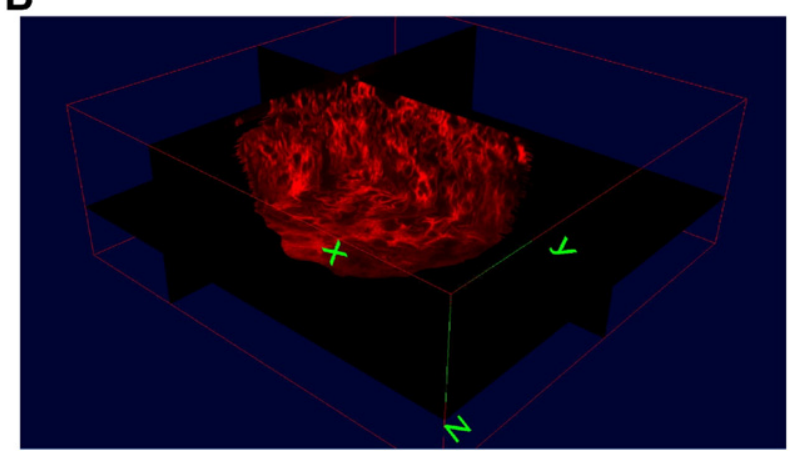

Figure 1. A: Three-dimensional stack reconstruction of z-section confocal images through a glomerulus without sclerosis to illustrate capillary branching pattern. B: Ortho-section cutaway of same glomerulus as in $\mathbf{A}$ to show branching patterns and architecture of glomerulus in $\mathrm{x}, \mathrm{y}$, and $\mathrm{z}$ axes. Capillary loops and basement membranes are visualized by the lectin lens culinaris (red). Original magnification, $\times 60$. 

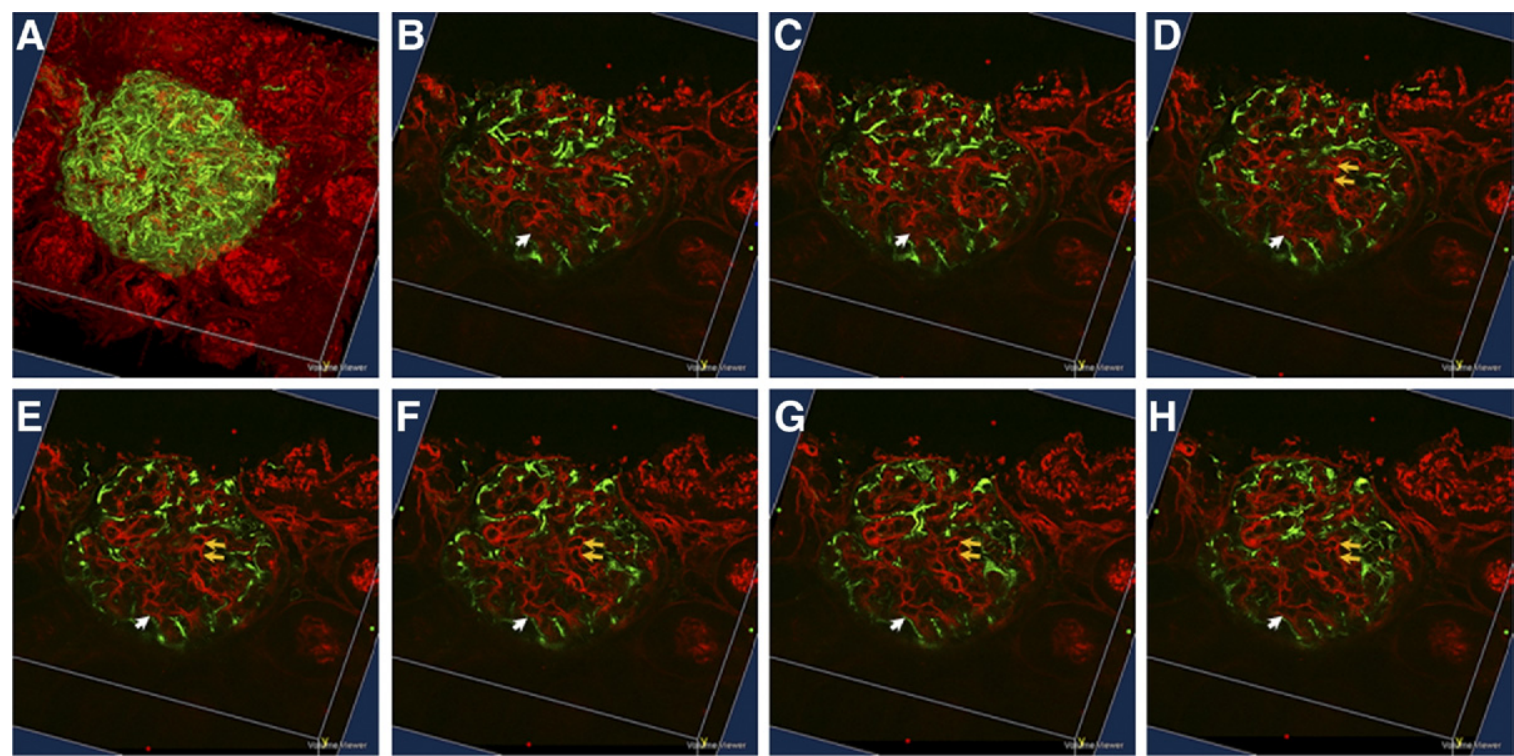

Figure 2. A-H: Three-dimensional stack reconstruction of z-section confocal images through a glomerulus without sclerosis to illustrate capillary branching pattern (red) and show podocytes (green) (A, same glomerulus as in Figure 1). Representative z-section confocal serial images are shown with representative branch points indicated with arrows in $\mathbf{B}-\mathbf{H}$. A single capillary lumen identified with a white arrow in $\mathbf{B}$ shows elongated profile and continuity with adjacent luminal segments in $\mathbf{H}$. Similarly, 2 separate capillary lumina indicated by yellow arrows in $\mathbf{D}$ show coalescence in $\mathbf{H}$, again demonstrating a branching point. Podocytes are stained with antibody to vimentin (green), and capillary loops and basement membranes visualized by the lectin lens culinaris (red). Original magnification, $\times 60$.

where $\mathrm{V}_{\text {glom }}$ is glomerular volume and 2 accounts for afferent and efferent arterioles of the glomerulus.

Graph theory analysis was used to further explore the topology of the glomerular capillary network sampled. Capillary branch points were identified on each z-section by overlaying adjacent sections in Adobe Photoshop (Adobe Systems Incorporated, San Jose, CA) to make comparisons. Each branch point was assigned a unique integer value as it was identified. To construct a topological map of the glomerular network, each numbered branch point was represented as a vertex on a graph. Capillaries connecting two branch points were depicted on the graph as edges. All connecting pairs of branch points represent the complete structure of capillary network assessed. As detailed above, this assessment included some complete tuft profiles, as well as some tufts where not all but most of the tuft could be adequately imaged. The complete set of vertex pairs comprises an edge set, which was entered into the Combinatorica package of Mathematica (Wolfram Research Incorporated, Champaign, IL) for calculation of graph invariants.

Results are expressed as mean \pm SEM. Statistical difference was assessed by single-factor variance (analysis of variance). Levene's test was used to test for the ho- mogeneity of variances. Post hoc comparisons were performed with least significant difference or Tamhane's T2 test, depending on the results of homogeneity test. $P<$ 0.05 was considered significant.

\section{Results}

Confocal z-sections were collected throughout the glomerular tufts, capturing most of the glomerular volume, as illustrated in a representative three-dimensional stack reconstruction in Figure 1, A and B. Examples of branch points of capillaries are illustrated in Figure $1 \mathrm{~B}$ with an ortho-section cutaway view of the stack and in serial sections in Figure 2. Glomeruli were analyzed in this fashion from nonsclerosed glomeruli in normal kidneys. We compared glomerular topology with that in segmentally sclerotic glomeruli from week 12 of untreated 5/6 nephrectomy rats with proven progression of sclerosis, as assessed in our previous study of these rats with analysis of sclerosis by light microscopy, and with that in glomeruli from rats with biopsy-proven regression of existing sclerosis induced by high-dose ARB treatment from week 8 to 12 after 5/6 nephrectomy (Figure 3). ${ }^{4}$
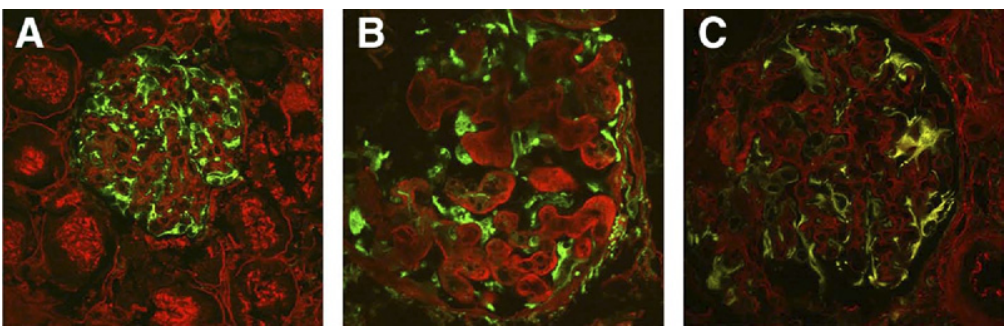

Figure 3. Confocal images of normal glomerulus (A), glomerulus with sclerosis (B), and glomerulus with ARB-induced regression of sclerosis $(\mathbf{C})$. Podocytes are stained with antibody to vimentin (green), and capillary loops and basement membranes are visualized by the lectin lens culinaris (red) Original magnification, $\times 60$. 
Table 1. Glomerular Characteristics in Normal Versus Progression and Regression of Sclerosis

\begin{tabular}{lccc}
\hline & Normal & Progression & Regression \\
\hline Glomerular volume, mean $\pm \mathrm{SEM}, \times 10^{-5} \mu \mathrm{m}^{-3}$ & $6.35 \pm 0.45$ & $10.1 \pm 1.56^{\star}$ & $11.6 \pm 2.33^{\star}$ \\
Capillary no. per glomerulus, mean $\pm \mathrm{SEM}$ & $115 \pm 18$ & $16 \pm 5^{\dagger}$ & $114 \pm 28$ \\
Length of capillaries per glomerulus, mean \pm SEM, mm & $10.02 \pm 0.23$ & $1.10 \pm 0.40^{\star}$ & $9.50 \pm 2.22$ \\
\hline
\end{tabular}

${ }^{*} P<0.01$ versus normal.

${ }^{\dagger} P<0.001$ versus normal or regression.

Thus, tissue comparisons were made from week 12 after $5 / 6$ nephrectomy in these selected rats with similar biopsy-proven progression versus those with regression of sclerosis. We then compared these glomeruli from rats with progression or regression to normal glomeruli from the kidneys removed at the time of 5/6 nephrectomy in these same rats. ${ }^{4} \mathrm{~A}$ total of 5709 confocal sections were obtained from 33 glomeruli from nine rats for analysis (11 glomeruli from each group).

Glomerular volume was markedly increased in untreated 5/6 nephrectomy rats at week 12 . In glomeruli from rats with regression of sclerosis at week 12 after $5 / 6$ nephrectomy induced by ARB initiated at week 8 , glomerular volume remained increased $(P<0.01$ for normal versus progression or regression, $P=$ nonsignificant for progression versus regression). However, further analysis of the composition of glomeruli with similar volume after 5/6 nephrectomy revealed significantly decreased capillary numbers, with corresponding decreased capillary length and surface area in progression versus regression (Table 1). Thus, glomeruli with regression have significant restoration of capillary segments, with increased capillary segment number and capillary length, compared with progressive sclerosis. In regression, these capillary measures are similar to normal glomeruli.

We next assessed the complexity of capillary branching patterns in each group by calculating graph invariants. Each z-stack was transformed into a graph (Figure 4). Graph order was calculated as a measure of total vertices in each graph. The mean order of normal glomeruli was $126.27 \pm 7.70$. The mean order of glomeruli from rats with sclerosis was reduced to $28.27 \pm 4.17(P<0.001)$, whereas the graph order for glomeruli from rats with regression was $75.00 \pm 6.23$, demonstrating an increase in capillary branch points in rats that had received ARB treatment and had regression compared with rats without treatment after 5/6 nephrectomy $(P<0.001)$ (Figure 5).

Graph size was calculated as the sum of the total number of edges in each graph, corresponding to the total number of capillary segments in each glomerulus. The mean graph size for normal glomeruli was $183.45 \pm 10.39$. Glomeruli from rats with progressive sclerosis displayed a significantly reduced graph size of $33.73 \pm 3.95(P<0.001)$. Regression led to a significantly increased graph size of $87.27 \pm 8.06(P<0.001)$, compared with glomeruli with progressive glomerulosclerosis (Figure 6). This increase in graph size after ARB treatment supports the concept that new capillaries can form in response to ARB, potentially replacing those lost during initial stages of glomerulosclerosis.

The root distance (RD) of each graph was calculated as the shortest path from source vertex to the sink vertex, corresponding to the smallest number of capillary branch points separating the afferent arteriole from the efferent arteriole in each glomerulus. For normal glomeruli, the RD was $10.63 \pm 1.03$. Glomeruli from rats with progression had an RD of $10.27 \pm 1.35$. Consistent with increased branch points along the path, the RD for glomeruli from rats with regression increased to $14.27 \pm 1.62(P<$ 0.001) (Figure 7A).

The complexity of the capillary network was analyzed by calculating RD/order for each graph (Figure 7B). An $\mathrm{RD} /$ order value of 1 reflects a linear path through the glomerulus. Disruption of individual capillary segments would critically affect such a network. RD/order values closer to 0 reflect increased complexity in the capillary network and thus less reliance on single pathways. Flow through a complex network is not significantly affected by the loss of a small number of individual capillary segments because many alternative pathways exist to traverse the network from "source to sink." Normal glomeruli had an RD/order value of $0.08 \pm 0.01$. Glomeruli from rats with sclerosis had a markedly increased RD/order value of $0.37 \pm 0.09$ ( $P<0.001)$, correlating with decreased complexity of branching. In contrast, glomeruli from rats with regression had a significantly reduced $\mathrm{RD} /$ order value of $0.19 \pm 0.02$, reflecting restoration toward the normal complexity of branching. Thus, overall, graph in-
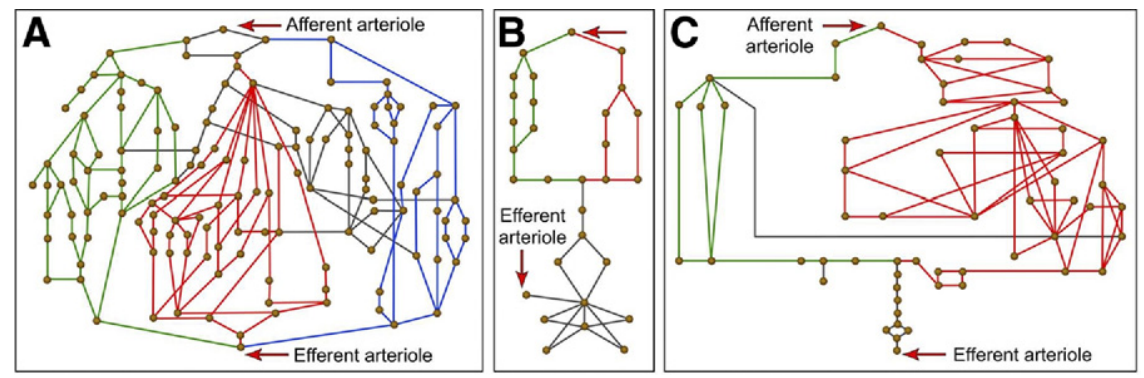

Figure 4. Graphs of topological networks for normal glomerulus (A), glomerulus with sclerosis (B), and glomerulus with ARB-induced regression of sclerosis (C). Separate lobes within each network form subgraphs indicated with different colors. Separate lobes are identified by interlobular connections that form minimal edge cuts 


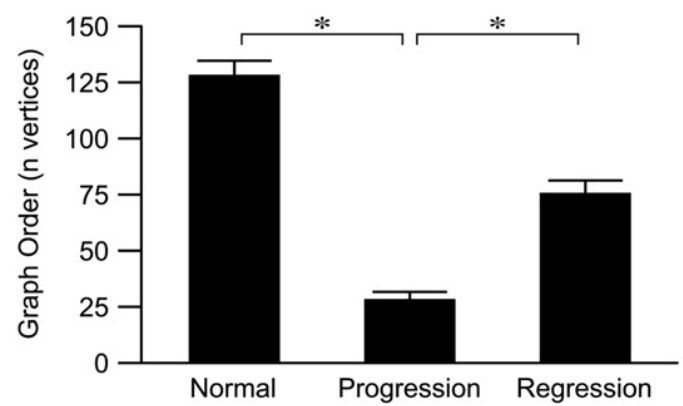

Figure 5. Graph order for normal glomeruli, glomeruli with sclerosis, and glomeruli with ARB-induced regression of sclerosis, indicating number of vertices $\left({ }^{*} P<0.001\right)$

variants for glomeruli from rats with regression displayed increased capillary branching compared with glomeruli from rats with progression. These rats with regression at week 12 induced by ARB treatment starting at 8 weeks after 5/6 nephrectomy had restoration of these capillary topologic measures to levels similar to that of normal.

\section{Discussion}

Our previous work and that of others have shown that regression of existing glomerulosclerosis can indeed occur. ${ }^{4,8-11,17-19}$ Studies by Adamczak et $\mathrm{al}^{9}$ have shown that delayed intervention with high-dose enalapril in the $5 / 6$ nephrectomy cautery model had parallel effects on glomerulosclerosis, tubulointerstitial, and vascular damage, with regression of existing lesions. Some previous studies have focused on a decreased area of sclerosis and decreased matrix accumulation as key mechanisms affecting regression. ${ }^{4,8,11}$ More recently, changes in glomerular cellular composition have also been investigated. In the studies by Adamczak et al, glomerular volume and podocyte number were not affected, but regression was associated with fewer cells within the mesangium. Our previous studies by light microscopy and electron microscopy in the 5/6 nephrectomy model interestingly showed that although regression of sclerosis was achieved in a subset of rats with inhibition of the renin-angiotensin-aldosterone system, podocyte injury remained. Thus, extensive foot process effacement was

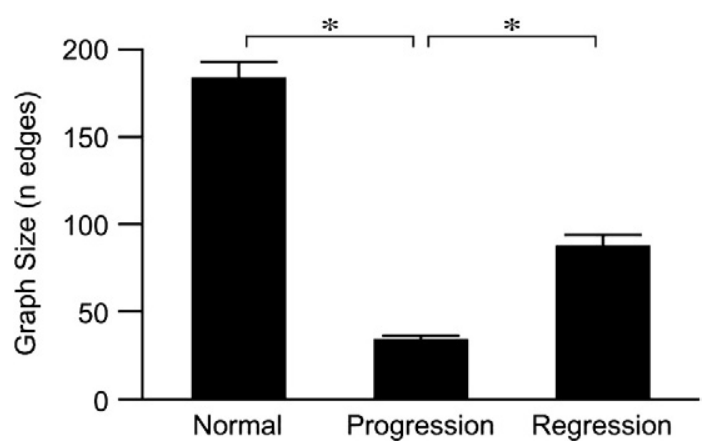

Figure 6. Graph size for normal glomeruli, glomeruli with sclerosis, and glomeruli with ARB-induced regression of sclerosis, indicating number of edges $\left({ }^{*} P<0.001\right)$
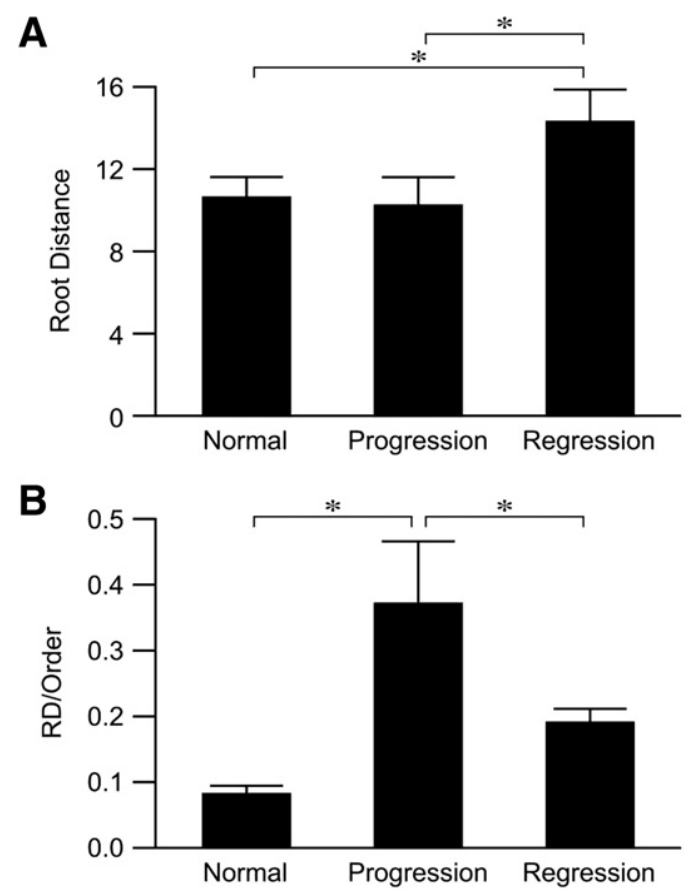

Figure 7. A: RD, the shortest path from source to sink vertex between afferent and efferent arterioles for normal glomeruli, glomeruli with sclerosis, and glomeruli with ARB-induced regression of sclerosis. B: RD/order values indicating the linearity of the graph for normal glomeruli, glomeruli with sclerosis, and glomeruli with ARB-induced regression of sclerosis. A RD/ order of 1 reflects a linear path $\left({ }^{*} P<0.001\right)$.

present at week 12 in untreated 5/6 nephrectomy rats, and partial foot process effacement remained even in animals that had achieved regression of sclerosis. ${ }^{20}$ The limited regenerative capability of the podocyte may underlie this divergence in time course of light microscopic improvement and restoration of normal podocyte architecture. We speculate that limitations of the podocyte may thus ultimately limit the potential of regression of glomerulosclerosis, unless specific therapies are identified that can specifically target increased regeneration of podocytes. Endothelial cells per glomerulus were decreased when regression was achieved in the studies by Adamczak et al. ${ }^{9}$ In additional studies by this group, the stereologic and topologic characteristics of glomerular capillary segments were assessed using standard morphometry techniques. Regression was associated with a decrease in mean glomerular volume, a decreased number of capillary segments per glomerulus, and a decreased total length of capillaries per glomerulus but without any significant change in the length of individual capillary segments and no change of numerical capillary density of the tuft. ${ }^{10}$

We then chose to expand on these previous approaches by studying branching patterns of glomerular topology by confocal analysis coupled with a novel graph theory analysis of the morphological complexity of the glomerulus in progressive disease versus regression states. Limitations of this approach include the technical limits on depth of glomeruli that can be adequately visualized and thus analyzed by this method. 
Thus, only the smaller glomeruli that were captured within the thick vibratome sections could be completely optically sectioned and visualized, with the branching point analysis of such glomeruli illustrated in Figure 1 and Figure 4. For larger glomeruli, analyses were based on a portion of the entire tuft. Of note, the analytical approaches applied are not contingent on entire tuft sampling. Similarly, assessments of any structural lesion and comparisons between groups are not dependent on sampling and assessment of the entire kidney three-dimensionally. Thus, we used nonbiased sampling based on morphometric principles and analyzed all sections with optimal image quality. This approach allows for further analysis and understanding of differences in the capillary topology in the treated rats with regression and those with progression compared with the normal rats.

Of note, traditional morphometric approaches do not account for the complexity of branching, a possible key event in glomerular angiogenesis. Thus, glomeruli with similar capillary length density (length per unit volume of glomerulus) might have different branching patterns, which in turn influences flow, pressures, and understanding of pathogenesis of glomerular architectural remodeling in sclerosis and its regression. Our current studies show that in this model of progressive glomerulosclerosis, glomeruli are large, but this larger than normal volume is primarily composed of sclerosis (ie, obliterated capillary loops due to increased matrix). Correspondingly, in sclerotic glomeruli, not surprisingly, there is a decreased number and length of capillary segments. Conversely, the large glomeruli observed when regression of sclerosis is induced by ARB in this model show increased capillary segment number and length, similar to those levels observed in normal. The variance in results from those reported by Adamczak et al may reflect in part differences in models. Our 5/6 nephrectomy model has more severe hypertension and more severe progressive sclerosis induced by ligation of renal artery branches, in contrast to removal of renal mass by cautery. ${ }^{9}$ In addition, we selected rats with similar biopsyproven regression and compared them with untreated remnant kidney rats with similar biopsy-proven progression; thus, these selected data are not necessarily representative of the more heterogeneous groups of all rats after $5 / 6$ nephrectomy, where lesions may be much more variable.

Recently, Remuzzi et $\mathrm{al}^{7}$ studied regression in aging male Munich Wistar Fromter rats, a spontaneous experimental model for progressive renal disease. Delayed ACEI treatment reduced glomerulosclerosis and interstitial fibrosis, but glomerular volume was not remarkably affected. However, the nonsclerotic glomerular volume increased significantly, suggesting formation of new glomerular capillary segments, consistent with our data. In the obese Zucker rats with sclerosis induced by uninephrectomy and a high-protein diet, either ACEI or ARB stopped progression of glomerulosclerosis. ${ }^{19}$ Both glomerular endothelial cell and podocyte numbers were restored by these interventions, with a trend toward increased glomerular capillary length density in treated rats, also consistent with our findings. However, the podocytes were not studied by electron microscopy, and thus it is unknown whether complete normalization of podocyte architecture was accomplished with those interventions. Overall, these results indicated that capillary branching can occur when remodeling is induced in a variety of injury settings.

To further explore the changes in glomerular topology underlying our observations of increased capillary segment number and length in regression, we used graph theory analysis. ${ }^{21-23}$ Measurement of graph order showed a significantly reduced number of capillary branch points in glomeruli after untreated 5/6 nephrectomy compared with normal glomeruli. In those $5 / 6$ nephrectomy animals treated with ARB that achieved regression of sclerosis, analyzed by light microscopic analysis, ${ }^{4}$ our current analysis shows that glomerular graph order was significantly increased. These data support that ARB treatment increases the complexity of the glomerular topology through the induction of new capillary segment branch points.

Conversely, graph size was reduced in glomeruli from rats with progressive glomerulosclerosis proven by our previous light microscopic assessment of sclerosis. The number of edges was significantly increased in glomeruli from rats with regression, indicating that $A R B$ treatment induces formation of new vessels. A measure of RD showed increased complexity of the capillary network in glomeruli from ARB-treated rats compared with glomeruli with progressive glomerulosclerosis from rats after 5/6 nephrectomy without treatment. The complexity of the glomerular network is critical for function. As the glomerular network decreases in complexity, each path becomes increasingly critical. The loss of a single path in such a glomerulus markedly disrupts flow between the afferent and efferent arterioles. As seen in progressive glomerulosclerosis, where glomeruli had an average RD/ order measure of 0.37 , there is a high dependence on individual pathways through the glomerulus. The continued loss of capillary segments during injury can ultimately lead to the loss of function altogether. Glomeruli from these ARB-treated rats that had regression had significant restoration of complexity (RD/order 0.19 versus 0.08 in normal rats), indicating that ARB treatment increases capillary branching with consequent increased complexity of the capillary network.

Our data are also in concert with recent in vitro studies where we showed that ARB can promote angiogenesis by affecting the interaction between podocytes and the glomerular endothelial cells. Podocytes are normally a key source of vascular endothelial-derived growth factor (VEGF), which is necessary for maintenance and growth of glomerular endothelial cells. When we injured podocytes in vitro, both VEGF and angiopoietin-1 were decreased, and the supernatant from injured podocytes did not stimulate glomerular endothelial cell growth. In contrast, when injured podocytes also were treated with ARB, VEGF and angiopoietin-1 were restored, and this supernatant supported increased growth and branching of glomerular endothelial cells. ${ }^{14}$ The current data support that ARB induces capillary growth in vivo as well. We 
hypothesize that this effect may be mediated in part by effects on podocytes.

Not all stages of sclerosis show equal remodeling capacity. Our previous work in this 5/6 nephrectomy model indeed supported that glomerular tufts with more than $50 \%$ sclerosis did not respond to high-dose ACEI but underwent progressive sclerosis. ${ }^{6}$ Observations from Remuzzi et al ${ }^{7}$ also strongly support the concept that glomeruli with extensive sclerosis do not benefit from ACEI. Our current data also support that regression involves coordinate remodeling and complex interactions of matrix remodeling with capillary growth. Previous in vitro studies have shown that receptor expression of mesangial cells is significantly affected by increased surrounding matrix with down-regulation of the platelet-derived growth factor receptor. ${ }^{24}$ Thus, as matrix is remodeled and its accumulation decreases, key receptors involved in angiogenesis may be altered, increasing potential for remodeling and capillary growth. Targeted interventions that affect both pathological matrix accumulation and promote capillary growth have, in theory, maximum potential to ameliorate and even regress existing sclerosis. Our data support that ARBs have such effects, although regression is not achievable in our model in all rats and all glomeruli. Studies by Zoja et a ${ }^{11}$ point to the benefits of multipronged intervention with, for example, RAS inhibition and statins to achieve regression. Whether additional combined therapy with further modulation of angiogenesis both directly and by restoring podocytes has even more potential to forestall CKD awaits further investigation.

\section{References}

1. Fogo AB: Can glomerulosclerosis be reversed? Nat Clin Pract Nephrol 2006, 2:290-291

2. Fogo AB: Progression versus regression of chronic kidney disease. Nephrol Dial Transplant 2006, 21:281-284

3. Fioretto P, Steffes MW, Sutherland DE, Goetz FC, Mauer M: Reversal of lesions of diabetic nephropathy after pancreas transplantation. N Engl J Med 1998, 339:69-75

4. Ma LJ, Nakamura S, Aldigier JC, Rossini M, Yang H, Liang X, Nakamura I, Marcantoni C, Fogo AB: Regression of glomerulosclerosis with high-dose angiotensin inhibition is linked to decreased plasminogen activator inhibitor-1. J Am Soc Nephrol 2005, 16:966-976

5. Fujihara CK, Velho M, Malheiros DM, Zatz R: An extremely high dose of losartan affords superior renoprotection in the remnant model. Kidney Int 2005, 67:1913-1924

6. Ikoma M, Kawamura T, Kakinuma Y, Fogo A, Ichikawa I: Cause of variable therapeutic efficiency of angiotensin converting enzyme inhibitor on glomerular lesions. Kidney Int 1991, 40:195-202
7. Remuzzi A, Gagliardini E, Sangalli F, Bonomelli M, Piccinelli M, Benigni A, Remuzzi G: ACE inhibition reduces glomerulosclerosis and regenerates glomerular tissue in a model of progressive renal disease. Kidney Int 2006, 69:1124-1130

8. Boffa JJ, Lu Y, Placier S, Stefanski A, Dussaule JC, Chatziantoniou C: Regression of renal vascular and glomerular fibrosis: role of angiotensin II receptor antagonism and matrix metalloproteinases. J Am Soc Nephrol 2003, 14:1132-1144

9. Adamczak M, Gross ML, Krtil J, Koch A, Tyralla K, Amann K, Ritz $\mathrm{E}$ : Reversal of glomerulosclerosis after high-dose enalapril treatment in subtotally nephrectomized rats. J Am Soc Nephrol 2003, 14:2833-2842

10. Adamczak M, Gross ML, Amann K, Ritz E: Reversal of glomerular lesions involves coordinated restructuring of glomerular microvasculature. J Am Soc Nephrol 2004, 15:3063-3072

11. Zoja C, Corna D, Camozzi D, Cattaneo D, Rottoli D, Batani C, Zanchi C, Abbate M, Remuzzi G: How to fully protect the kidney in a severe model of progressive nephropathy: a multidrug approach. J Am Soc Nephrol 2002, 13:2898-2908

12. Fogo AB: New capillary growth: a contributor to regression of sclerosis? Curr Opin Nephrol Hypertens 2005, 14:201-203

13. Eddy AA, Fogo AB: Plasminogen activator inhibitor-1 in chronic kidney disease: evidence and mechanisms of action. J Am Soc Nephrol 2006, 11:2999-3012

14. Liang XB, Ma LJ, Naito T, Wang Y, Madaio M, Zent R, Pozzi A, Fogo AB: Angiotensin type 1 receptor blocker restores podocyte potential to promote glomerular endothelial cell growth. J Am Soc Nephrol 2006, 17:1886-1895

15. Yoshida Y, Fogo A, Ichikawa I: Glomerular hemodynamic changes vs. hypertrophy in experimental glomerular sclerosis. Kidney Int 1989, 35:654-660

16. Yoshida $Y$, Kawamura T, Ikoma M, Fogo A, Ichikawa I: Effects of antihypertensive drugs on glomerular morphology. Kidney Int 1989 , 36:626-635

17. Piecha G, Koleganova N, Gross ML, Geldyyev A, Adamczak M, Ritz $\mathrm{E}$ : Regression of glomerulosclerosis in subtotally nephrectomized rats: effects of monotherapy with losartan, spironolactone, and their combination. Am J Physiol Renal Physiol 2008, 295:F137-144

18. Marinides GN, Groggel GC, Cohen AH, Border WA: Enalapril and low protein reverse chronic puromycin aminonucleoside nephropathy. Kidney Int 1990, 37:749-757

19. SebekovÁ K, Lill M, Boor P, Heidland A, Amann K: Functional and partial morphological regression of established renal injury in the obese Zucker rat by blockade of the renin-angiotensin system. Am J Nephrol 2008, 29:164-170

20. Aldigier JC, Kanjanbuch T, Ma LJ, Brown NJ, Fogo AB: Regression of existing glomerulosclerosis by inhibition of aldosterone. J Am Soc Nephrol 2005, 16:3306-3314

21. Bacallao RL, Yu W, Dunn KW, Phillips CL: Novel light microscopy imaging techniques in nephrology. Curr Opin Nephrol Hypertens 2003, 12:455-461

22. Wahl EM, Quintas LV, Lurie LL, Gargano ML: A graph theory analysis of renal glomerular microvascular networks. Microvasc Res 2004, 67:223-230

23. Phillips $\mathrm{CL}$, Gattone VH 2nd, Bonsib SM: Imaging glomeruli in renal biopsy specimens. Nephron Physiol 2006, 103:75-81

24. Marx M, Daniel TO, Kashgarian M, Madri JA: Spatial organization of the extracellular matrix modulates the expression of PDGF-receptor subunits in mesangial cells. Kidney Int 1993, 43:1027-1041 